\section{An Ordinary Multilayer Fruit Paper Bag Supplying Device for Fruit Bagging}

\author{
Hongmei Xia ${ }^{1}$, Wenbin Zhen, Dongyang Chen, and Wen Zeng \\ College of Engineering, South China Agricultural University, 483 Wushan \\ Road, Guangzhou, Guangdong 510642, China
}

Additional index words. fruit planting, fruit bagging machine, automatic bag supplying device, open hand, design

\begin{abstract}
Taking out and opening the ordinary multilayer fruit paper bag for fruit bagging is labor intensive, costly, not efficient, and potentially dangerous to the operator's health. There is a high demand to develop a mechanical device for the operation in Chinese orchards. A novel supplying device based on the manual operated fruit bag case was proposed. The open hand of the supplying device operates like a farmer's hand that can continuously take out a multilayer fruit paper bag one by one, and open it fully from its inside. Mechanism configuration and dimension parameter of the open hand were designed based on preliminary tests. The operation functionality of the supplying device prototype at different driving trajectories and speed was investigated in the study. The laboratory experimental data indicated that driving trajectory was an extremely significant factor for efficiently taking out and opening the fruit bag without sliding off and damage. Driving speed had a beneficial effect on reducing supplying time. With the synchronous driving trajectory and allowable high moving speed, the developed supplying device could achieve more than $90 \%$ opening success rate and less than 2 second opening time. The study showed the potential of the developed mechanical supplying device for fruit bagging with ordinary multilayer fruit paper bags.
\end{abstract}

Preharvest fruit bagging has been a popular physical protection technique in several countries, such as China, Japan, and the United States. It is an effective way to improve fruit appearance, control pests and diseases, and reduce pesticide residues (Amarante et al., 2002; Hofman et al., 1997; Huang et al., 2009; Sharma and Sanikommu, 2018). China is the largest fruit planting and output area in the world ( $\mathrm{Wu}, 2017)$. Lower quality and poor market competitiveness are concerning problems for Chinese fruit growers (Zhang, 2017). Fruit bagging is a favorable solution to raise fruit prices and increase economic benefits for Chinese fruit farmers (Guo, 2017; Li et al., 2016; Matsumoto et al., 2018; Sharma et al., 2014; Zhou et al., 2019). Compared with the singlelayer fruit paper bag, the multilayer fruit paper bag has been used more widely, because it provides larger lightproof property and lower temperature for better coloration of fruits (Zhao and $\mathrm{Wu}, 2015)$. The fruit

\footnotetext{
Received for publication 26 Apr. 2019. Accepted for publication 22 May 2019.

We acknowledge the financial support of the subproject (Grant No. 2017YFD0701301) of the National 13th Five-year Key Research and Development Plan Project (Grant No. 2017YFD0701300), the Science and Technology Planning Project of Guangdong Province (Grant No. 2017A020208052), and the National Science Project of China (Grant No. 51005080).

${ }^{1}$ Corresponding author. E-mail: xhm_scau@scau. edu.cn.

This is an open access article distributed under the CC BY-NC-ND license (http://creativecommons. org/licenses/by-nc-nd/4.0/).
}

bagging process with ordinary multilayer paper bag consists of the following major steps: taking out one bag, opening the bag, putting the young fruit into the bag, and sealing the upper end of the bag. Because of the complicated operation process, automatic fruit bagging machinery suitable for the ordinary multilayer fruit paper bag is still in the research phase.

Research on mechanical fruit bagging machinery for paper fruit bags dates back to the 1990s (Monta et al., 1995a). Monta et al.

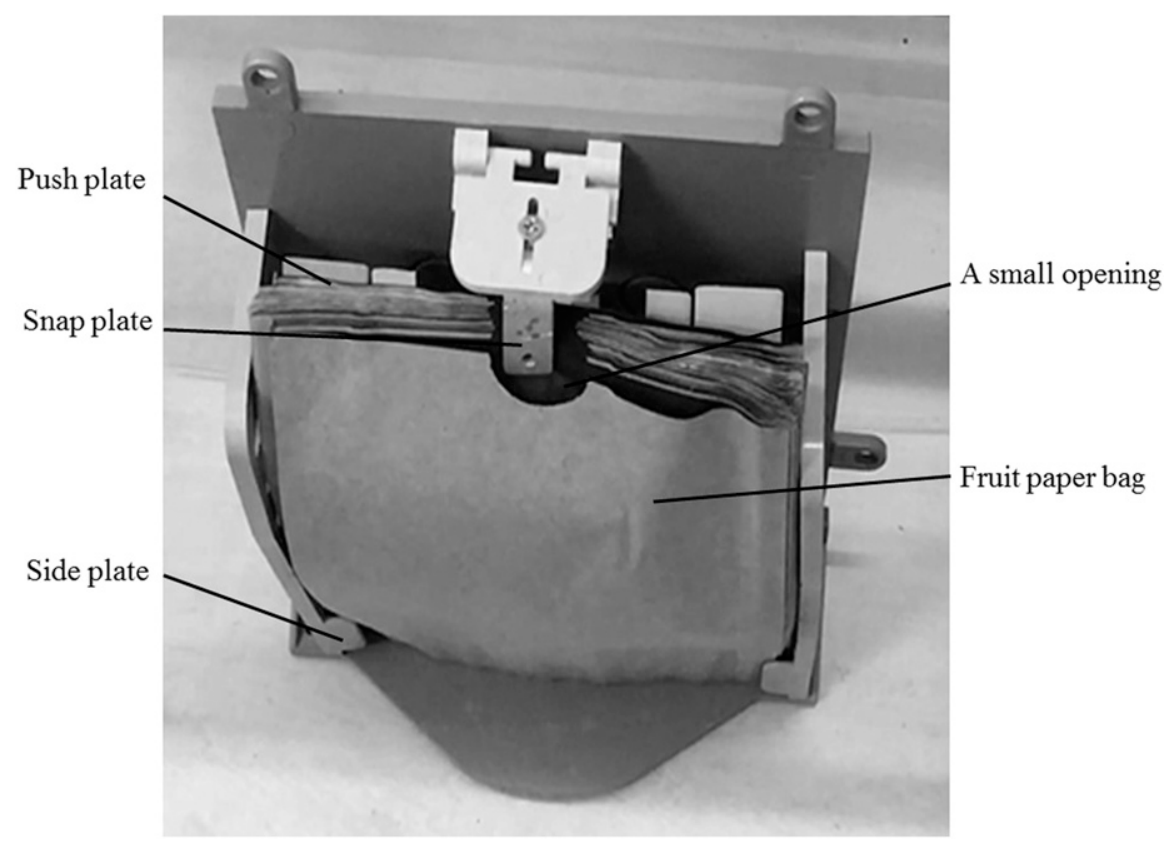

Fig. 1. Fruit bag case. (1995b and Li et al. (2011) developed a bagging end-effector that could open or close the bag by pressing two leaf springs, which were set face to face at the upper end of the bag. Hua et al. (2016) developed a portable, low-cost semiautomatic fruit bagging apparatus. The bag was fit with rubber bands along the rim and four plastic rings on its four corners, and could be delivered by a screwed pipe and slid out individually. $\mathrm{Xu}$ et al. (2007) developed an automatic bag-opening mechanism that could separate piled bags one by one by using a couple of vacuum sucking pans, and enlarge the bag inside maximally through arc fingers. Four wires were set on the upper end of the bag. Leng (2015) invented a bag-opening device that could separate two sides of the bag by magnet attraction. Many metal wires were set on the front side of the bag. Zhang (2016) designed an apple bagging apparatus that opened the bag by two pneumatic suction cups.

Most previous studies were based on robot technology (Fang, 2011; Ge, 2005; Wang, 2016; Wen et al., 2017), and the structure of the fruit paper bag has been improved to simplify the bagging operation. Those research achievements have not been widely applied in China because of cost considerations. According to the special terrain feature of Chinese orchards, a cheap auxiliary fruit bagging device is more desirable and applicable than the expensive automatic fruit bagging robot (Wang et al., 2018). A type of manually operated fruit bag case has been widely used in Chinese orchards, as shown in Fig. 1. The fruit paper bags are set in the fruit bag case by the spring-driven push plate and two bottom side plates. The snap plate on the upper side of the fruit bag case presses against the crescent of the outermost bag, so that the upper end of the outermost 
bag forms a small opening. In the process of artificial fruit bagging, farmers hang the fruit bag case on their right side. They first hold their right hand fingers together to form a taper, then insert the held fingers into the outermost bag, and finally pull their hand outward to take the outmost bag out of the fruit bag case, and extend the held fingers to open the bag fully. The device is suitable for an ordinary single-layer paper bag, but not the ordinary multilayer fruit paper bag. As the inner bag of the multilayer paper bag is every thin and easy to adhere, taking out one bag and opening it fully by hand is timeconsuming, labor intensive, costly, and it is easy to bring out a broken bag. In addition, it is possibly dangerous to the farmers' health because their hands need contact with the inner bag repeatedly. Therefore, there is a demand for a mechanized method of taking out and opening the ordinary multilayer fruit paper bag for fruit bagging.

Existing methods of taking out and opening the fruit paper bag mainly include vacuum adsorption, magnetic absorption, and pressing a leaf spring on the upper end of the bag. As different layers of the ordinary multilayer bag were generally bonded at the side edge and some local points (Zhang, 2009,2012 ), those methods are not favorable to fully open the inner bag to avoid bag broken problem. To mechanize the taking out and opening operation for the ordinary multilayer fruit paper bag, the primary goal of the study was to develop a bag supplying device based on the previously described manually operated fruit bag case. The specific objectives of the study were to 1 ) propose a mechanism configuration scheme of the bag supplying device and determine its critical structure parameters; and 2) investigate functionality and practicality of the developed mechanical bag supplying device through laboratory experimentation.

\section{Materials and Methods}

Working method of the bag supplying device. The designed prototype diagram of the bag supplying device is shown in Fig. 2 (Xia et al., 2018). The fruit bag case is the same as that shown in Fig. 1. The open hand works as a farmer's hand. The preopen fan

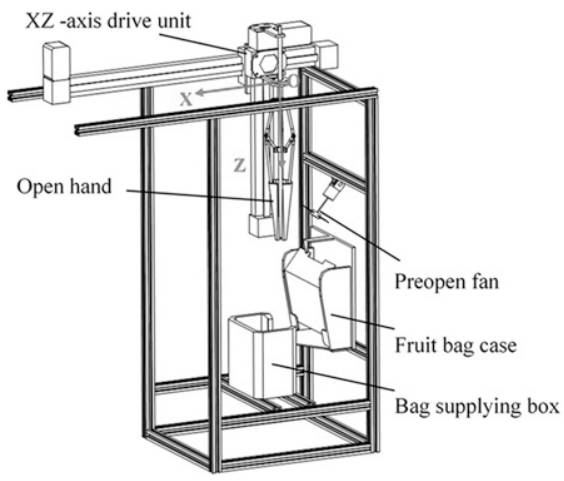

Fig. 2. Prototype diagram of the bag supplying device. can blow the outmost bag in the fruit bag case to a certain open size for easily inserting the open hand. The open hand driven by the XZaxis drive unit first moves to the work origin $\mathrm{O}$ with initial folded state, where the symmetrical center of the open hand is consistent with the center of the outmost bag in the fruit bag case. Then the open hand moves down into a certain depth of the outermost bag, pulls the outermost bag out of the fruit bag case, extends the bag fully to an unrecoverable deformation, and takes the opened bag to the bag supplying box. Finally, the open hand moves back to its work origin position again for the next bag.

Design of the open hand. The open hand is the key component of the bag supplying device, and has three symmetrical opening rods, as shown in Fig. 3. The slider $\mathrm{X}$ is fixed connect with the guiding rod. The slider $\mathrm{Z}$ can move along the guiding rod to drive the three opening rods open or withdraw. The connection relationships between the slider $\mathrm{Z}$ and the opening rod, the linkage rod and the opening rod, the linkage rod and the limit slider are all hinge constraints. The limit stopper is fixed connect at the bottom of the guiding rod. Relative sliding of the limit slider relative to the guiding rod is a redundant $\mathrm{df}$, which would be eliminated if the limit slider is restricted by the limit stopper.

Mechanism configuration state of the open hand with one opening rod in the fruit bag supplying process is shown in Fig. 4. At the initial state, as shown in Fig. 4A, the open hand stays at its original position, which is above the center of the fruit paper bag, the end of the opening rod gathers around the guiding rod, and the limit slider stays above the limit stopper. For entering into the inside of the fruit paper bag, the slider $\mathrm{Z}$ and limit slider move downward a stroke of $s_{z i}$ along the guiding rod synchronously, until the limit slider is blocked by the limit stopper, as shown in Fig. 4B. For taking out and opening the fruit bag, the slider X moves a stroke of $s_{x}$, the slider $\mathrm{Z}$ moves down a stroke of $s_{z o}$. In this process, the limit slider remains at the bottom of the guiding rod and the opening rod extends to open the bag, as shown in Fig. 4C.

The main parameters of the open hand are marked as shown in Fig. 4. The initial distance of the limit stopper above the center of the paper fruit bag is $\mathrm{H}_{0}$, as shown in Fig. 4A. On the opening rod, the AB segment represents as $l_{1}$, BD segment represents as $l_{3}$, the angle between $\mathrm{AB}$ and $\mathrm{BD}$ represents as $\delta$. The length of the linkage rod $\mathrm{BC}$ represents as $l_{2}$. In Fig. $4 \mathrm{~A}$ and $\mathrm{B}$, the initial angle of the opening rod relative to the guiding rod is $\alpha_{0}$, the initial angle of the linkage rod relative to the guiding rod is $\beta_{0}$, the initial distance of the slider $Z$ relative to the limit stopper is $h$, and the initial force transmission angle of the point $\mathrm{B}$ is $\gamma_{B} \mathrm{~h}$. As shown in Fig. $4 \mathrm{C}$, at the opened state, the angle between the opening rod and the guiding rod is $\alpha$, the angle between the linkage rod and the guiding rod is $\beta$, the distance between the endpoint $\mathrm{D}$ of the opening rod and the bottom of the fruit bag is $\mathrm{H}$, the opened radius of the endpoint $\mathrm{D}$ on the opening rod is $r$. A smaller $\gamma_{B}$ is favorable for keeping the initial folded state of the opening rod, whereas a larger $\gamma_{B}$ is valuable for extending the opening rod to the opened state. According to the force transmission requirements of the open hand at different states, here $\gamma_{B}$ takes $45^{\circ}$. For reducing $l_{3}$ and overall size of the open hand, there is: $\alpha_{0}<\beta_{0}$.

If $\beta_{0}=2 \alpha_{0}$, then $\alpha_{0}=15^{\circ}, \beta_{0}=30^{\circ}$.

According to Fig. 4, we can obtain the following relationships:

$$
\begin{gathered}
l_{1} \sin \alpha-l_{2} \sin \beta=0 \\
l_{1} \sin \alpha_{0}=l_{2} \sin \beta_{0}=l_{3} \sin \left(\delta-\alpha_{0}\right) \\
l_{1} \sin \alpha+l_{3} \sin (\alpha-\delta)=r
\end{gathered}
$$

For fully opening a typical type of doublelayer fruit paper bag with a size of $170 \mathrm{~mm}$ width and $210 \mathrm{~mm}$ height, based on manual preliminary experiment, $H_{0}$ sets as $20 \mathrm{~mm}$, and $H$ sets as $10 \mathrm{~mm}, r$ sets as $57 \mathrm{~mm}$ due to maximum open requirement of the open hand, and $l_{3}$ takes as $237 \mathrm{~mm}$, there exists:

$$
l_{3} \cdot \cos (\alpha-\delta)-l_{2} \cdot \cos \beta=220
$$

Calculated from Eq. [1] to Eq. [4], there are:

$$
\begin{gathered}
l_{1}=55 \mathrm{~mm} \\
l_{2}=29 \mathrm{~mm} \\
\delta=18^{\circ} \\
\alpha=26^{\circ} \\
\beta=59^{\circ}
\end{gathered}
$$

The opening rod is a key part that directly contacts and deforms the fruit paper bag. To ensure the bottom of the open hand can easily insert into the fruit bag, and achieve a good deformation effect, a circular arc extension surface was designed based on the preliminary tests, as shown in Fig. 5. For easily entering the bag without breaking the inner layer, and maintaining adequate friction to avoid the bag sliding off, proper material of the extension surface is a concerning problem. Contact effects of different extension surface materials were evaluated, which showed that more elastic material, like rubber, had a large contact friction coefficient with the inner layer of the fruit bag, which would lead to the high possibility of large deformation or even rupture of the inner paper bag. Material with higher rigidity, like aluminum alloy, had a small contact friction coefficient with the inner layer of the fruit bag, which would enhance the possibility of the fruit bag sliding off the extension surface. Polylactide (PLA) material was chosen as the extension surface material based on the preliminary comparison experiments. 


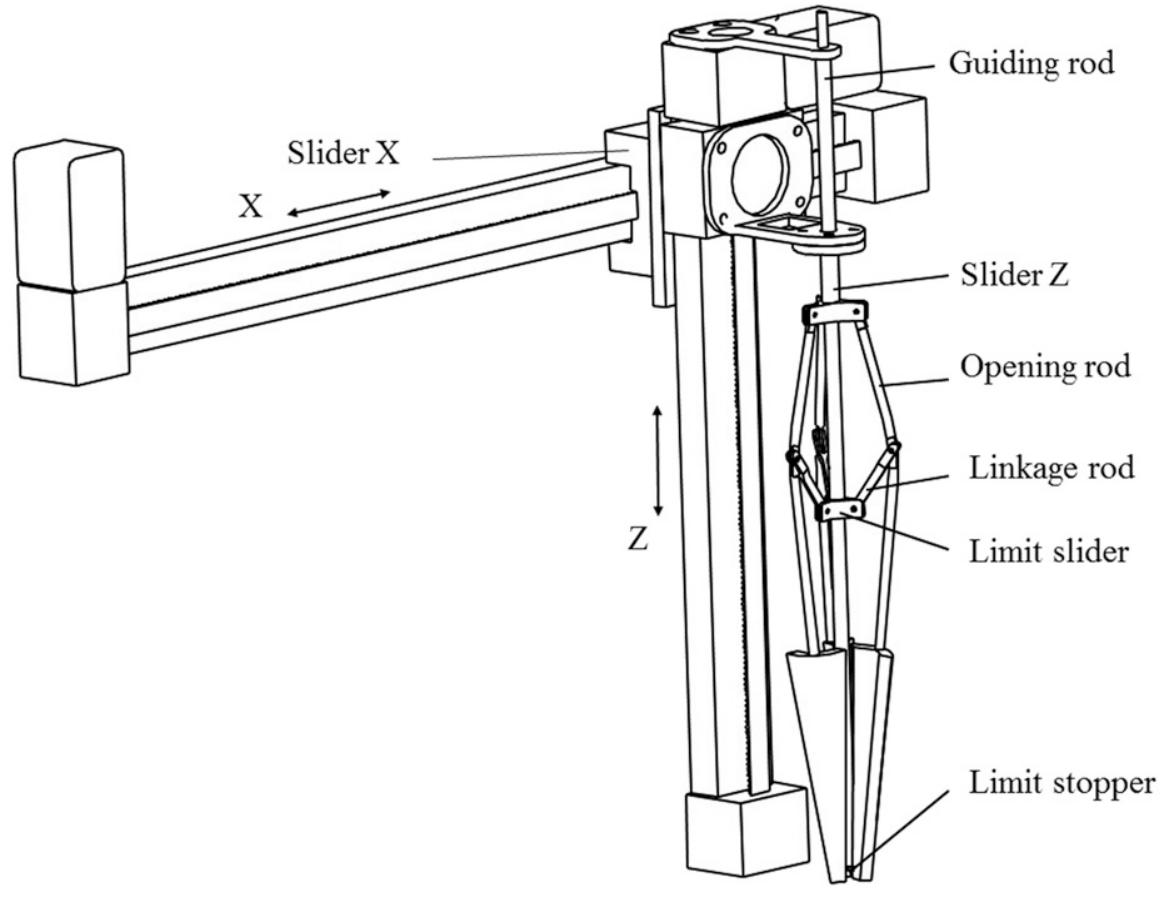

Fig. 3. Prototype diagram of the open hand.

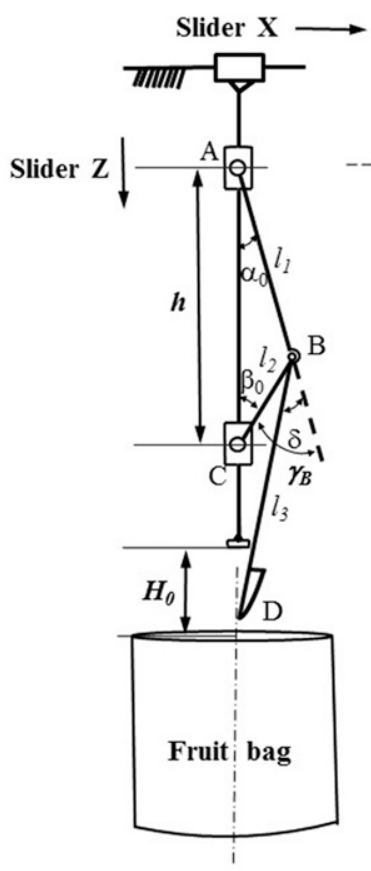

A

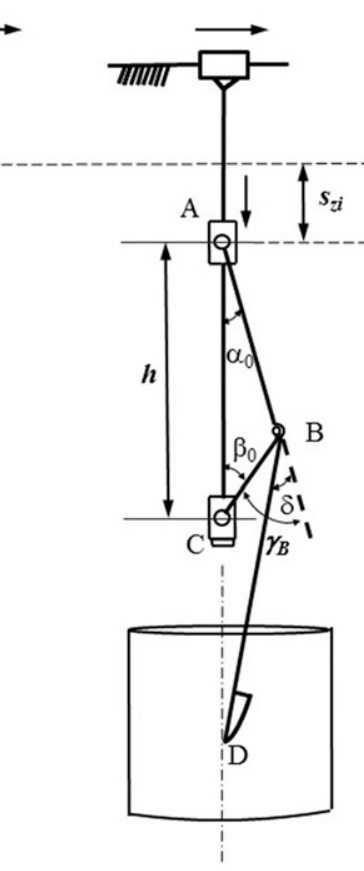

B

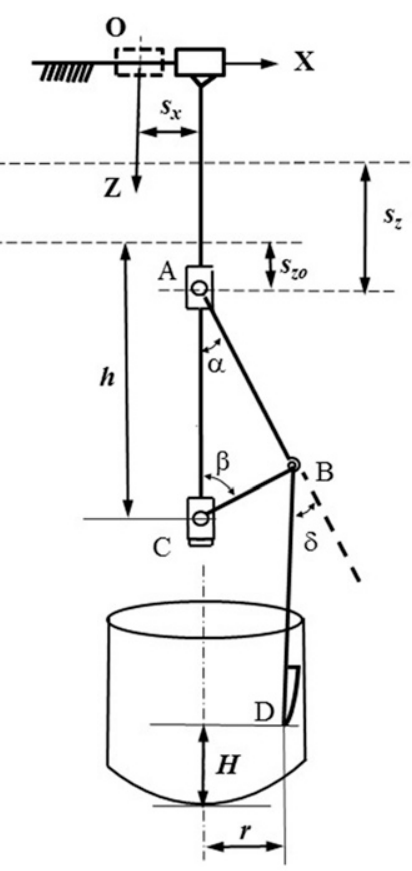

C
Fig. 4. Configuration state change of the open hand with one opening rod. (A) Initial state of the open hand at the original position; (B) the open hand entering into the fruit paper bag with initial folded state; and (C) the open hand extending state.
Bag supplying device validation. A laboratory prototype of the supplying device was fabricated based on previous design, as shown in Fig. 6. The XZ-axis drive unit consisted of two sets of 57BYGH748 stepper motor and M542-05 motor driver, which could accurately slide $\mathrm{X}$ to a maximum stroke of $500 \mathrm{~mm}$, and slide $\mathrm{Z}$ to a maximum stroke of $300 \mathrm{~mm}$. It was commanded by a motion controller (TC55 tor (FC2541; Telesky, Shenzhen, China) from 0 to $15000 \mathrm{r} / \mathrm{min}$. At the speed of $8000 \mathrm{r} / \mathrm{min}$, the blown transverse preopen distance of the bag was about $45 \mathrm{~mm}$, which was enough for the open hand easily entering into the bag. The programmable smart timer (SM5D, SUHED, Shenzhen, China) was used for recording the operation time. The work origin of the open hand was set to be the same with the origin of the XZ-axis drive unit. A type of double-layer fruit paper bag with $170 \mathrm{~mm}$ width and $210 \mathrm{~mm}$ height was selected for the test, as shown in Fig. 6. It is suitable for fruit bagging agronomic requirements of $\approx 40$ to 60 -mm-diameter young fruit, such as mango, pear, apple, or peach.

The whole motion trajectory of the open hand includes two stages. The first stage, (1), is entering into the inside of the fruit bag with the initial folded state, and the second stage, (2), is taking out and opening the fruit bag, as shown in Fig. 7. Based on the preliminary test, $200 \mathrm{~mm}$ of $s_{z i}$ is suitable for the open hand entering into enough depth of the fruit bag, $60 \mathrm{~mm}$ of $s_{x}$ is the minimum stroke for extending the multilayer fruit paper bag out of the fruit bag case, and $20 \mathrm{~mm}$ of $s_{z o}$ is proper for fully opening the fruit bag from the inside. For the second stage, (2), the motion trajectory can be designed as a sloping line, circular, and polyline. Through programming at the motion controller, the open hand was driven at a different trajectory and speed.

For investigating the influence of the driving trajectory and speed, three types of trajectories and six levels of driving speed were determined as the test factors, as shown in Table 1. Three times repeated test for the two factors were conducted. The bag supplying effects was evaluated by the supplying success rate $\eta(\%)$ and supplying time $t_{s}(\mathrm{~s})$. The supplying success rate $\eta(\%)$ was defined by Eq. [5], and the supplying time $t_{s}(\mathrm{~s})$ was determined by Eq. [6]

$$
\begin{gathered}
\eta=\frac{n_{s}}{n} \times 100 \% \\
t_{s}=\frac{T}{n} \times 100 \%
\end{gathered}
$$

where $n_{s}$ is the number of the fruit paper bags that were taken out of the fruit bag case and opened to an unrecoverable deformation without damage or sliding off in one experiment; $T$ is the bag supplying time recorded by the smart time in one experiment; and $n$ is the tested total number of bags in one experiment.

In each experiment, 30 fruit paper bags were continuously taken out and opened in one experiment. The average fruit bag supplying success rate $\eta(\%)$ and supplying time $t_{s}(\mathrm{~s})$ at different factor levels were compared by analysis of variance at the significance level of $P<0.05$ (Duncan's method).

four-axis controller). The TYD-89 fruit bag case suitable for $\approx 130$ to $175-\mathrm{mm}$ width and $\approx 160$ to 225 -mm height fruit paper bag was adopted. Based on the preliminary test, installation angle of the preopen fan revolve center relative to the horizontal plane was set at $\approx 45^{\circ}$. The preopen fan was driven by a 130-2295-38 DC vane motor, the speed of which could be adjusted by the PWM DC motor speed regula-

\section{Results and Analysis}

The mean supplying success rate curves are shown in Fig. 8. With increase of the driving speed, the supplying success rate decreased correspondingly. When the driving speed grew from $25 \mathrm{~mm} / \mathrm{s}$ to $400 \mathrm{~mm} / \mathrm{s}$, the 


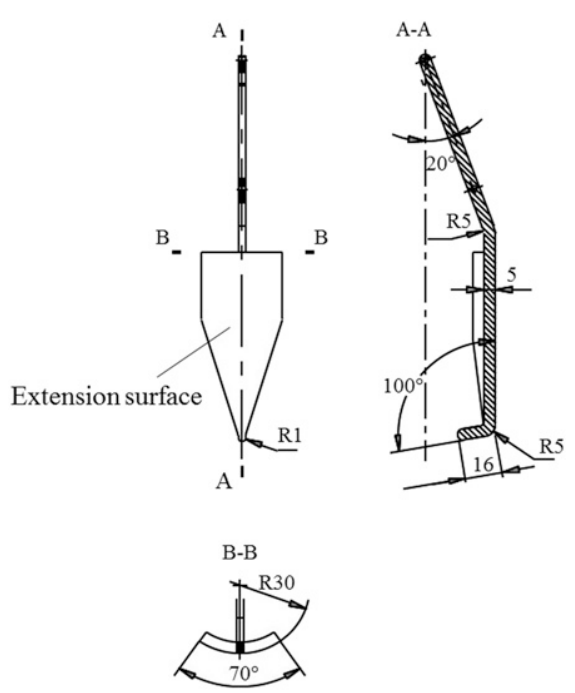

Fig. 5. Construction of the opening rod.

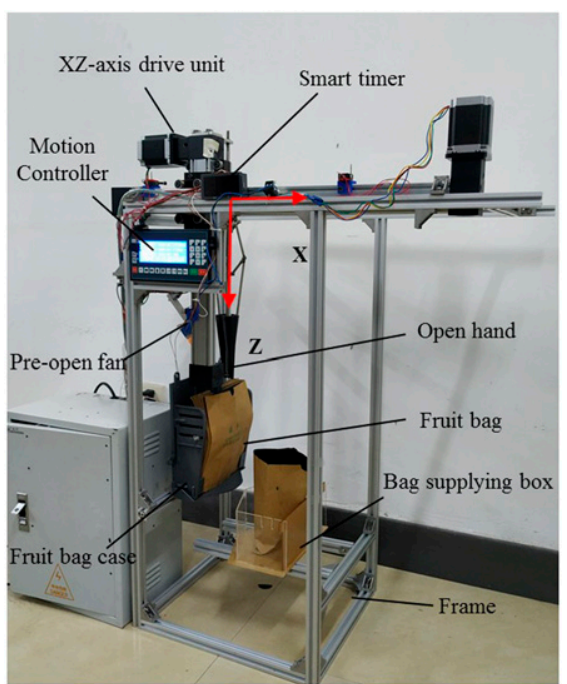

Fig. 6. Laboratory prototype of the supplying device.

supplying success rate reduced from $97.0 \%$ to $91.1 \%$ for the sloping line trajectory, from $97.8 \%$ to $87.8 \%$ for the circular trajectory, and from $77.8 \%$ to $71.11 \%$ for the polyline trajectory. Variance analysis results illustrated the influence of driving speed on the supplying success rate for each trajectory was insignificant. This might mainly attribute to the flexible and large nonlinear deformation characteristic of the fruit paper bag during the supplying process. It implies that driving speed does not have significant influence on the equivalent stress distribution and overall deformation of the fruit paper bag.

Main effect analysis on the mean supplying success rate is shown in Fig. 9. It indicated that polyline trajectory resulted in the lowest mean percentage of $73.45 \%$, whereas the sloping line and circular obtained 95.35\% and $90.56 \%$, respectively. The statistical analysis revealed supplying success rate of the polyline trajectory was significantly lower than that of the sloping line and circular trajectory, whereas there was no significant

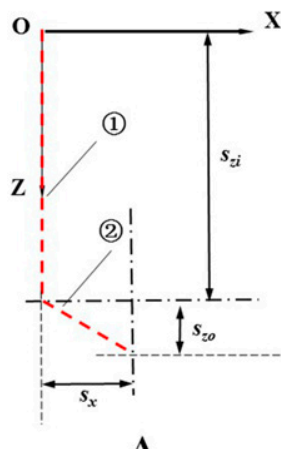

A

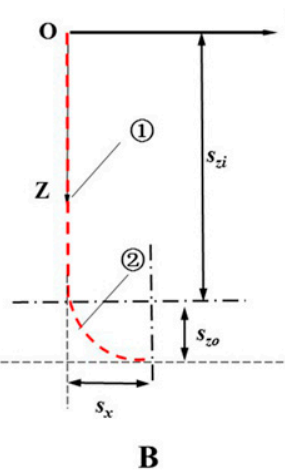

$\mathrm{x}$

र

Fig. 7. Motion trajectory. (A) Sloping line trajectory for the second stage; (B) circular trajectory for the second stage; and $(\mathbf{C})$ polyline trajectory for the second stage.

Table 1. Test factor and level.

\begin{tabular}{llc}
\hline & \multicolumn{2}{c}{ Factor } \\
\cline { 2 - 3 } Level & Trajectory & Speed $(\mathrm{mm} / \mathrm{s})$ \\
\hline 1 & Sloping line & 25 \\
2 & Circular & 100 \\
3 & Polyline & 175 \\
4 & & 250 \\
5 & & 325 \\
6 & & 400 \\
\hline
\end{tabular}

difference between the sloping line and circular trajectory. It was found from the supplying process that the fruit bag slipping off the open hand without being opened timely was the most failure phenomenon. For the sloping line and circular trajectory, the slider $\mathrm{X}$ and slider $\mathrm{Z}$ of the open hand were driven by the XZdriving unit synchronously. For the polyline trajectory, the slider $\mathrm{X}$ was first driven to a certain stroke, then the slider $\mathrm{Z}$ was driven to a certain stroke later. It indicated the synchronous drive could contribute to higher supplying success rate than the single axis independent drive method.

The mean supplying time curves are shown in Fig. 10. They indicated the higher driving speed is beneficial for reducing the supplying time. When the driving speed increased from $25 \mathrm{~mm} / \mathrm{s}$ to $400 \mathrm{~mm} / \mathrm{s}$, the supplying time decreased from $9.15 \mathrm{~s}$ to $1.47 \mathrm{~s}$ with the polyline trajectory, from $8.49 \mathrm{~s}$ to $0.84 \mathrm{~s}$ with the sloping line trajectory, and from $7.76 \mathrm{~s}$ to $0.72 \mathrm{~s}$ with the circular trajectory. The statistical analysis indicated driving speed was an extremely significant influence factor on supplying time for each trajectory, although when the driving speed was more than $200 \mathrm{~mm} / \mathrm{s}$, variation of the supplying time for each driving trajectory is not obvious. When the driving speed rose from $200 \mathrm{~mm} / \mathrm{s}$ to $400 \mathrm{~mm} /$ $\mathrm{s}$, the supplying time reduced from $1.04 \mathrm{~s}$ to $0.84 \mathrm{~s}$ for the sloping line trajectory, from 1.81 $\mathrm{s}$ to $1.47 \mathrm{~s}$ for the polyline trajectory, and from $1.09 \mathrm{~s}$ to $0.72 \mathrm{~s}$ for the circular trajectory. In addition, it was found that when the driving speed was higher than $300 \mathrm{~mm} / \mathrm{s}$, there was excessive vibration of the open device.

The main effect analysis on the supplying time is shown in Fig. 11. It indicated the polyline trajectory spent the largest average supplying time of $3.31 \mathrm{~s}$, whereas the sloping line and circular used $2.34 \mathrm{~s}$ and $2.17 \mathrm{~s}$ individually. The statistical results indicated supplying time of the polyline trajectory was significantly larger than that of the sloping line and circular trajectories, whereas there was no significant difference between the sloping line and circular trajectories.

Overall, this analysis indicated that the sloping line and circular trajectories are optimal for the high supplying success rate and less supplying time. For avoiding mechanical vibration of the supplying device, and achieving high efficiency, the ideal driving speed is $\approx 175 \mathrm{~mm} / \mathrm{s}$ to $250 \mathrm{~mm} / \mathrm{s}$. With the preferable driving trajectory and speed, the developed supplying device can achieve more than $90 \%$ supplying success rate and less than $2 \mathrm{~s}$ supplying time in the laboratory environment. Except sliding off the open hand, there appeared to be little tearing problem on the bottom bonding area of the paper fruit bag in the test. This problem mainly came from large deviation between initial center of the paper fruit bag and symmetrical center of the open hand, which leads to nonuniform stress and deformation of the fruit paper bag during the supplying process. Therefore, further improvement study will be conducted to enhance the stability of the supplying device, and achieve a high center position precision when the open hand is inserted into the fruit paper bag. In addition, the automatic supplying device will be mounted onto a field wagon and tested in the orchard.

\section{Conclusion}

Fruit bagging with an ordinary multilayer paper bag includes complicated operation steps. Most previous studies on automatic fruit bagging machines were based on robotic technology for improved fruit paper bag. Those methods are costly and suitable for large-scale fruit planting. In Chinese orchards, cheap auxiliary fruit bagging apparatus for manual operation is more desirable. Thus, a novel mechanical supplying device for ordinary multilayer fruit paper bag that can automatically pick out one bag and open it fully from its inside was developed and tested in the study. Key parameters and structure of the device were designed based 


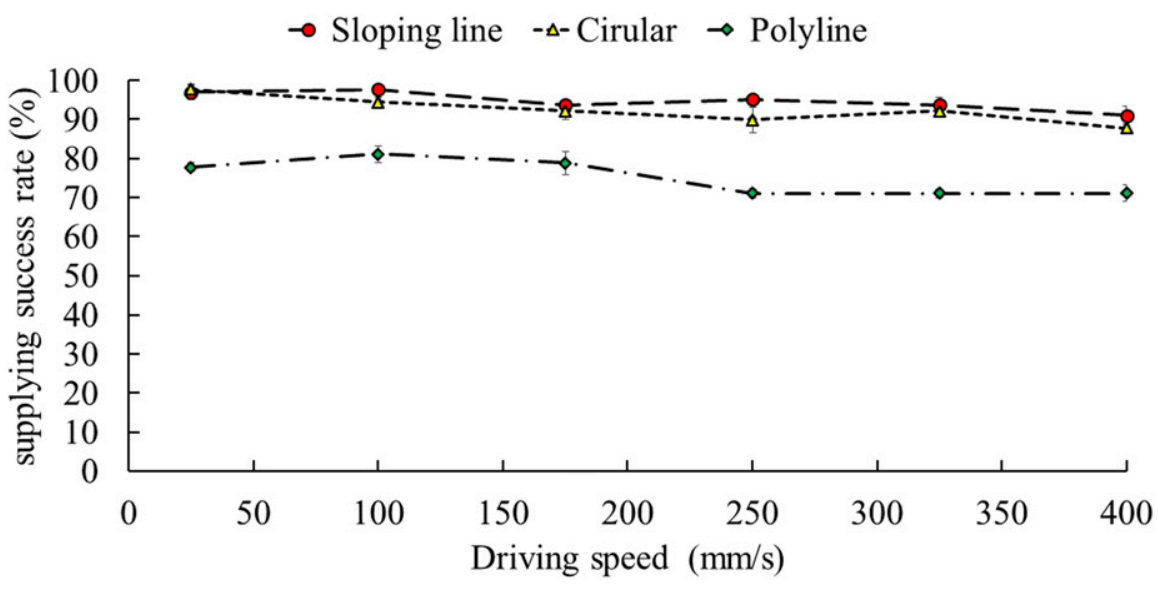

Fig. 8. Supplying success rate curves.

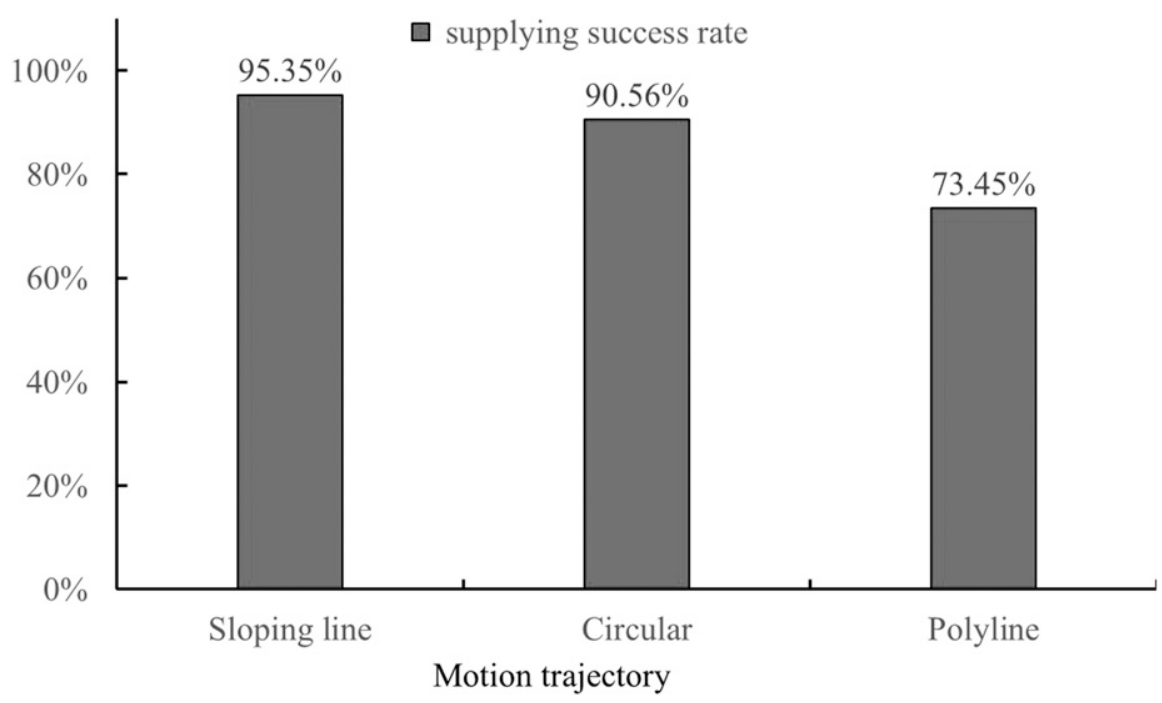

Fig. 9. Main effect histogram for supplying success rate.

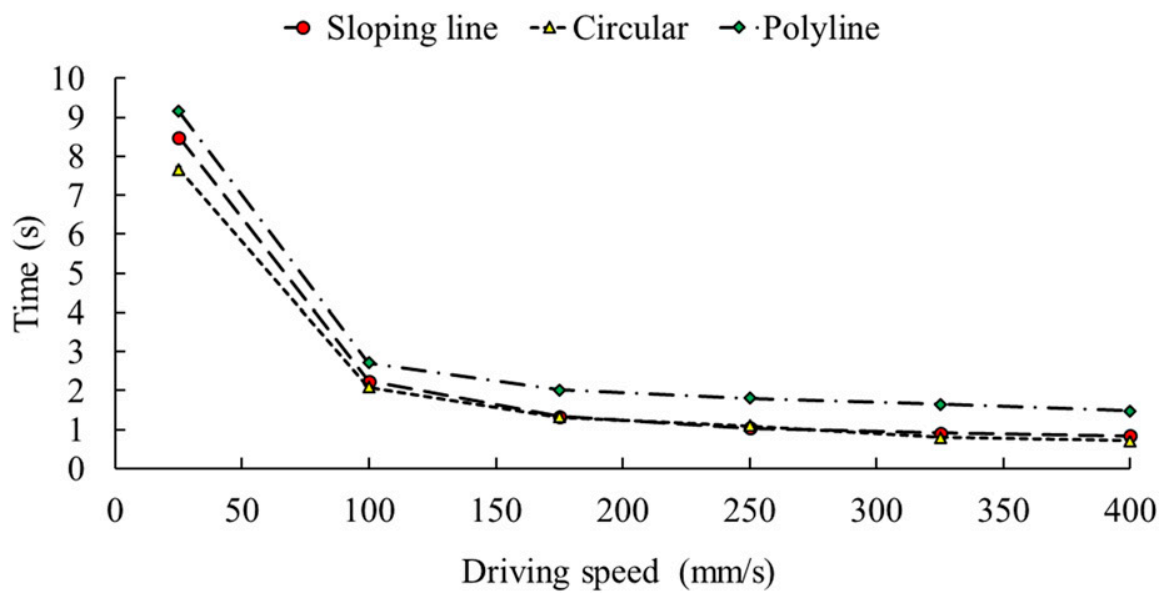

Fig. 10. Average supplying time curves.

on the preliminary test. A set of functionality verification experiments were conducted in the laboratory. The test results showed that the driving trajectory is an extremely significant factor for successful supplying of the bag without damage or sliding off problems,

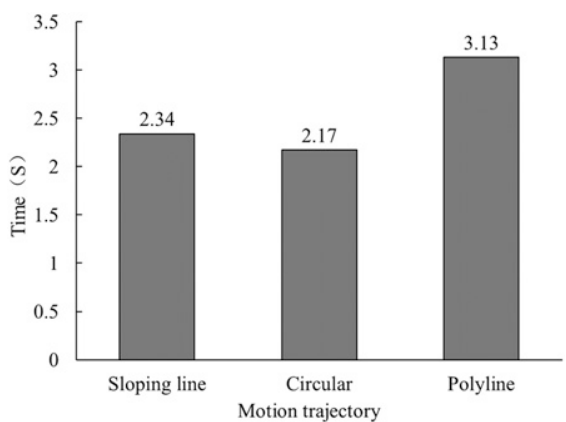

Fig. 11. Main effect histogram for average supplying time.

success rate. Bag supplying time highly depends on the driving speed, $175 \mathrm{~mm} / \mathrm{s}$ to 250 $\mathrm{mm} / \mathrm{s}$ were suitable for the supplying device running smoothly, and achieved less than 2-s supplying time, although influence of the driving speed on the supplying success rate is not significant.

The study showed the developed supplying device can take out and open the ordinary multilayer fruit paper bag from its inside at the same time, and keep the inner and outer layers of the multilayer fruit paper bag a uniform opening, which is conducive to improving the work efficiency and success rate for fruit bag supplying. It has good feasibility and applicability for supplying opened ordinary multilayer fruit paper bags continuously for the manual bagging operation. For further enhancing its supplying success rate, its structure stability and driving precision would be improved in the later study.

\section{Literature Cited}

Amarante, C., N.H. Banks, and S. Max. 2002. Effect of preharvest bagging on fruit quality and postharvest physiology of pears (Pyrus communis). N. Z. J. Crop Hort. Sci. 30(2):99-107.

Fang, O. 2011. Key technology research of fruit bagging machinery. Zhejiang Sci-Tech University, Hangzhou, MS Thesis.

Ge, X. 2005. Study on semi-automatic robot for apple bagging. China Agriculture University, Beijing, MS Thesis.

Guo, Y. 2017. Main technical measures for producing high quality apples - Evaluation of fruit bagging. Agr. Dev. Equipment. (04):157.

Hofman, P. J., L. G. Smith, D. C. Joyce, G. I. Johnson, and G. F. Meiburg. 1997. Bagging of mango (Mangifera indica cv. 'Keitt') fruit influences fruit quality and mineral composition. Postharvest Biol. Technol. 12(1):83-91.

Hua, Y., B. Yang, X.G. Zhou, J. Zhao, and L. Li. 2016. A novel progressively delivered fruit bagging apparatus. J. Appl. Hort. 18(2):123-127.

Huang, C., B. Yu, Y. Teng, J. Su, Q. Shu, Z. Cheng, and L. Zeng. 2009. Effects of fruit bagging on coloring and related physiology, and qualities of red Chinese sand pears during fruit maturation. Scientia Hort. 121(2):149-158.

Leng, K. 2015. A pocket opening device on young fruit bagging machine. P.R.C. Patent 201520164291.8. Filed 23 Mar. 2015. Issued 22 July 2015.

Li, W., L. Zhao, W. Wang, and Z. Sun. 2011. A grape bagging manipulator. P.R.C. Patent 201110161246.3. Filed 15 June 2011. Issued 26 Oct. 2011.

HortScience Vol. 54(9) September 2019 whereas its influence on the supplying time is insignificant. Compared with the single axi driving trajectory as polyline, the synchronous drive trajectories like sloping line an circular resulted in fewer problems of sliding off and achieved more than $90 \%$ supplying 
Li, Y., X. Qi, M. Lin, Z. Li, and J. Fang. 2016. Effect of bagging on fruit pigmentation in two types of red-fleshed kiwifruit. J. Fruit Sci. 33(12):14921501.

Matsumoto, K., T. Kobayashi, T. Kougo, T. Fujita, S. Sato, and T. Moriguchi. 2018. Prevention of new cork spot-like physiological disorder in 'Kurenainoyume' Apples by pre-harvest fruit bagging. Hort. J. 87(2):174-183.

Monta, M., N. Kondo, and Y. Shibano. 1995a. Agricultural robot in grape production system. Proc. 1995 IEEE Int. Conf. Robotics Automation. 3:2504-2509.

Monta, M., N. Kondo, Y. Shibano, and K. Mohri. 1995b. End-effectors for agricultural robot to work in vineyard. Acta Hort. 399(29):247-254.

Sharma, R.R., S.V.R. Reddy, and M.J. Jhalegar. 2014. Pre-harvest fruit bagging: A useful approach for plant protection and improved postharvest fruit quality-a review. J Pomology Hort. Sci. 89(2):101-113.

Sharma, R.R. and V.R. Sanikommu. 2018. Preharvest fruit bagging for better protection and postharvest quality of horticultural produce, $\mathrm{p}$. 455-489. In: M.W. Siddiqui (eds.). Preharvest modulation of postharvest fruit and vegetable quality. Academic Press, San Diego, CA.

Wang, S. 2016. The development design and research of a fruit bagging robot. Yanshan Univ., Qinghuangdao, MS Thesis.

Wang, Y., Y. Zhang, Y. Pu, J. Zhang, and F. Wang. 2018. Design of a new fruit tree bagging machine. IOP Conf. Ser. Mater. Sci. Eng. 452(4):042099.

Wen, Y., Z. Wang, Y. Wan, and J. He. 2017. Review on development of fruit bagging device. J. Chinese Agr. Mechanization 38(6):104-108.

$\mathrm{Wu}, \mathrm{J} .2017$. Further optimization and upgrading of fruit production and consumption structure. Farm Produce Mkt. Weekly (16):29-30.

Xia, H., J. Xia, W. Zhen, W. Zeng, B. Huang, H. Huang, D. Chen, and Q. Chen. 2018. Extracting and opening device and method for single layer or multi-layer paper fruit bagging. P.R.C. Patent CN201711364510. Filed 18 Dec. 2017. Issued 29 June 2018.
Xu, L., X. Ge, and T. Zhang. 2007. Automatic unfolding-open hand of fruit bag. Trans. CSAE 23(4):138-143.

Zhang, H. 2009. The research of technics and device in molding of fruit bag engine. Agricultural University of Hebei, Baoding, MS Thesis.

Zhang, H. 2012. Department of agriculture apple fruit bags industry standards. Yantai Fruits (04):49-50.

Zhang, H. 2017. Changing trend of fruit consumption structure in China based on import and export data. Yantai Fruits (2):6-7.

Zhang, M. 2016. Design of an apple fruit automatic bagging machine. Technol. Wind (14):113114.

Zhao, J. and J. Wu. 2015. China's fruit market and its prospect for 2020. Agr. Outlook 11(05):24 28.

Zhou, H., Z. Yu, and Z. Ye. 2019. Effect of bagging duration on peach fruit peel color and key protein changes based on iTRAQ quantitation. Scientia Hort. 246:217-226. 